BMJ Open Diabetes

Research \& Care

\section{Effects of D-allulose on glucose tolerance and insulin response to a standard oral sucrose load: results of a prospective, randomized, crossover study}

To cite: Franchi F, Yaranov DM, Rollini F, et al. Effects of Dallulose on glucose tolerance and insulin response to a standard oral sucrose load: results of a prospective, randomized, crossover study. BMJ Open Diab Res Care 2021;9:e001939. doi:10.1136/ bmjdrc-2020-001939

- Supplemental material is published online only. To view, please visit the journal online (http://dx.doi.org/10.1136/ bmjdrc-2020-001939).

Received 5 0ctober 2020 Revised 19 January 2021 Accepted 11 February 2021

Check for updates

(c) Author(s) (or their employer(s)) 2021. Re-use permitted under CC BY-NC. No commercial re-use. See rights and permissions. Published by BMJ.

${ }^{1}$ Department of Medicine, University of Florida College of Medicine-Jacksonville, Jacksonville, Florida, USA ${ }^{2}$ Matsutani Chemical Industry Co. Ltd, Itami, Japan ${ }^{3}$ Kagawa University, Takamatsu, Japan

Correspondence to Dr Francesco Franchi; francesco.franchi@jax.ufl.edu

\section{ABSTRACT}

Introduction Current dietary guidelines recommend limiting sugar intake for the prevention of diabetes mellitus (DM). Reduction in sugar intake may require sugar substitutes. Among these, D-allulose is a non-calorie rare monosaccharide with $70 \%$ sweetness of sucrose, which has shown anti-DM effects in Asian populations. However, there is limited data on the effects of D-allulose in other populations, including Westerners.

Research design and methods This was a prospective, randomized, double-blind, placebo-controlled, crossover study conducted in 30 subjects without DM. Study participants were given a standard oral $(50 \mathrm{~g})$ sucrose load and randomized to placebo or escalating doses of D-allulose $(2.5,5.0,7.5,10.0 \mathrm{~g})$. Subjects crossed-over to the alternate study treatment after 7-14 days of wash out. Plasma glucose and insulin levels were measured at five time points: before and at 30, 60, 90 and 120 min after ingestion.

Results D-allulose was associated with a dose-dependent reduction of plasma glucose at 30 min compared with placebo. In particular, glucose was significantly lower with the $7.5 \mathrm{~g}$ (mean difference: $11 ; 95 \% \mathrm{Cl} 3$ to $19 ; \mathrm{p}=0.005$ ) and $10 \mathrm{~g}$ (mean difference: $12 ; 95 \% \mathrm{Cl} 4$ to $20 ; \mathrm{p}=0.002$ ) doses. Although glucose was not reduced at the other time points, there was a dose-dependent reduction in glucose excursion compared with placebo, which was significant with the $10 \mathrm{~g}$ dose $(p=0.023)$. Accordingly, at $30 \mathrm{~min} \mathrm{D}$-allulose was associated with a trend towards lower insulin levels compared with placebo, which was significant with the 10 $g$ dose (mean difference: $14 ; 95 \% \mathrm{Cl} 4$ to $25 ; \mathrm{p}=0.006$ ). $\mathrm{D}$-allulose did not reduce insulin at any other time point, but there was a significant dose-dependent reduction in insulin excursion compared with placebo $(\mathrm{p}=0.028)$, which was significant with the $10 \mathrm{~g}$ dose $(\mathrm{p}=0.002)$.

Conclusions This is the largest study assessing the effects of D-allulose in Westerners demonstrating an early dose-dependent reduction in plasma glucose and insulin levels as well as decreased postprandial glucose and insulin excursion in subjects without DM. These pilot observations set the basis for large-scale investigations to support the anti-DM effects of D-allulose.

Trial registration number NCT02714413.

\section{Significance of this study}

What is already known about this subject?

D-allulose is defined one of the rare sugars, which has been shown in animal and clinical studies, conducted mostly in Asian populations, to have postprandial plasma glucose suppressive effects with antiobesity and antidiabetic effects.

What are the new findings?

- Our study showed that D-allulose administered in addition to a standard sucrose load led to a dosedependent reduction of plasma glucose at $30 \mathrm{~min}$ compared with placebo with a dose-dependent reduction in plasma glucose excursion.

- The effects of D-allulose on insulin levels and insulin excursion were similar to those on plasma glucose.

D-allulose effects appeared to be consistent in both white and African-American subjects.

- The administration of escalating doses of D-allulose appeared to be safe.

How might these results change the focus of research or clinical practice?

- The results of our investigation can set the basis for the potential use of D-allulose in a population where reduction of added sugar is of paramount importance.

The present study suggests that adding $5-10 \mathrm{~g}$ of $\mathrm{D}$-allulose to $50 \mathrm{~g}$ of sugar is sufficient to reduce the postprandial glycemia attributed to sugar.

- This ratio of $\mathrm{D}$-allulose to sugar content is consistent with the composition of the currently marketed rare sugar syrup and is well tolerated.

- Future studies should explore more in depth the underlying mechanisms by which $\mathrm{D}$-allulose reduces glucose and insulin levels as well as its long-term effects, including its potential to impact clinically meaningful outcomes. 


\section{INTRODUCTION}

Although dieting is the cornerstone of weight management, the optimal mix of diet to facilitate weight loss and maximize the health benefits remains controversial. With the rapid increase in the prevalence of obesity worldwide, there is urgency in identifying an effective and safe diet for body weight reduction. ${ }^{1}$ While low fat diet has been the traditional cornerstone of the dietary guidelines of various national and international societies, there is increased popular attraction to low carbohydrate diets. $^{2-6}$ More importantly, there is growing evidence for potential health benefits of low glycemic foods, and glycemic load is the best quantitative variable that can be correlated with clinical outcomes, including coronary artery disease, breast cancer and type 2 diabetes mellitus (DM). ${ }^{7}$ Notably, a substantial portion of glycemic load in western diets is attributed to added caloric sweeteners in the diet, which has been steadily increasing over the last four decades and associated with development of metabolic syndrome and type $2 \mathrm{DM}^{89}$

Individuals in the USA consume a substantial proportion of their total energy as added sugars. Unlike most other carbohydrates, added sugars alone contribute no nutrients other than energy and are easily modifiable. The mechanism through which the dysmetabolic effects of carbohydrates occur is not completely understood. Studies suggest that these effects could be mediated by fructose, a monosaccharide comprising $50 \%$ of table sugar. Fructose has been shown to increase de novo lipogenesis in the liver, hepatic triglyceride synthesis and increase secretion of very low-density lipoproteins. Fructose also appears to decrease the peripheral clearance of lipids. ${ }^{10}$ These findings support the current dietary guidelines that encourage consumers to limit their intake of added sugar. ${ }^{10-13}$

Drastic reductions in the consumption of sugar may necessitate the use of sugar substitutes. Reduced calorie sweeteners and non-nutritive sweeteners currently approved by the US Food and Drug Administration (FDA) have limitations and have not proven to confer metabolic benefits. This underscores the need for a sugar substitute that is safe, palatable and has favorable effects on energy metabolism and overall glucose homeostasis. ${ }^{14} 15$

D-allulose, also referred to in the literature as D-psicose, a C-3 epimer of D-fructose, is defined one of the rare sugars since it is rarely found in nature. It is present in small quantities in commercial mixtures of D-glucose and D-fructose obtained from hydrolysis of sucrose or isomerization of D-glucose. ${ }^{16} \mathrm{D}$-allulose is a non-calorie monosaccharide, which has approximately $70 \%$ sweetness of sucrose. Animal and clinical studies have shown postprandial plasma glucose suppressive effects with antiobesity and antidiabetic effects. ${ }^{17-27}$ However, to date, clinical studies have been conducted mostly in Asian populations and there are still insufficient data to confirm the efficacy of pure D-allulose in Western populations, including white and African-American. The aim of this study was to address the efficacy of D-allulose in reducing postprandial blood glucose level in a random sample of a US population.

\section{METHODS}

\section{Study design and participants}

This was a single-center, prospective, randomized, doubleblind, placebo-controlled crossover study evaluating the effects of pure D-allulose ingestion on the glucose and insulin response to a standardized oral glucose load in a non-selected US population. The study was performed at the University of Florida Health-Jacksonville (Jacksonville, Florida, USA). Subjects between 18 and 70 years of age, a body mass index (BMI) between 20 an $40 \mathrm{~kg}$ / $\mathrm{m}^{2}$, without a diagnosis of DM and a hemoglobin Alc (HbA1c) $<5.8 \%$ were recruited from the community as well as from clinically stable ambulatory patients. Details on study inclusion and exclusion criteria are provided in the online supplemental material 1.

After providing written informed consent, subjects meeting inclusion/exclusion criteria were given a food diary along with instructions on how this should be filled, and were scheduled to return to complete screening within 7 days. Prior to the continuation of screening, study subjects completed overnight fasting of at least 8 hours, after which screening labs were collected. Eligible subjects were asked to bring their 72-hour food diary for evaluation by a dietitian. An individualized diet plan was provided, which consisted of $50 \%-65 \%$ of the subject's caloric needs from carbohydrates, which was followed throughout the whole course of the study. Subjects were asked to come back for a total of five treatment visits and were randomly assigned to sequentially receive varying amounts of D-allulose $(2.5,5,7.5,10 \mathrm{~g})$, or placebo (water) given in a random order in addition to a standardized oral sucrose load of $50 \mathrm{~g}$. In order to limit the possibility of carry-over effect, patients were randomized to different treatment sequences. In each sequence, all four doses were present only one time and were administered in a different order. A washout period of 1-2 weeks was done between each visit. At each visit, all administered sugars (sucrose plus D-allulose/placebo), which were not distinguishable by flavor, were dissolved in $300 \mathrm{~mL}$ of water to be consumed within $10 \mathrm{~min}$. Randomized treatment was prepared by a research pharmacist, in order to maintain double-blinding. Blood draws were obtained at five time points for measurement of blood glucose $(\mathrm{mg} / \mathrm{dL})$ and insulin levels $(\mu \mathrm{U} / \mathrm{L})$ : before (after overnight fasting) and 30, 60, 90 and 120 min after sucrose plus D-allulose/ placebo. All blood tests were carried out at Central Labs of UF Health Jacksonville using the blood collection tubes recommended by these labs. At each treatment visit, body weight and height were measured and BMI calculated, and food diary was reviewed to ensure adherence to the provided diet plan. A flow diagram of the study design is illustrated in figure 1. 


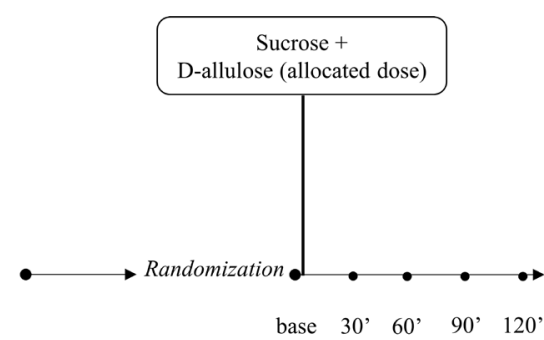

Visit 1

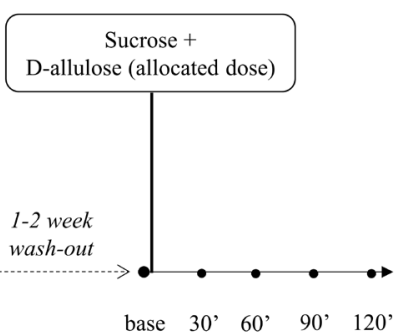

Visit 2

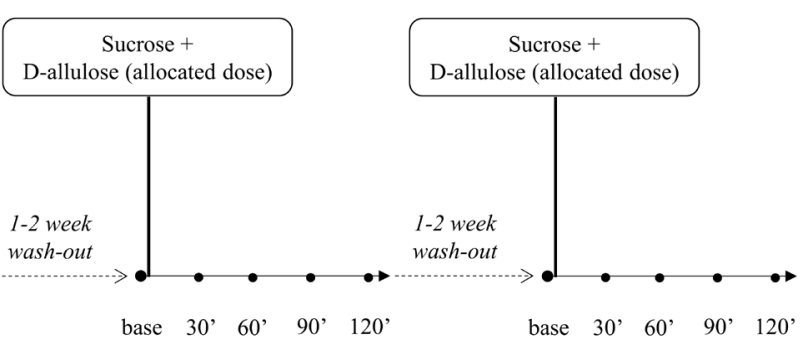

Visit 3

Visit 4

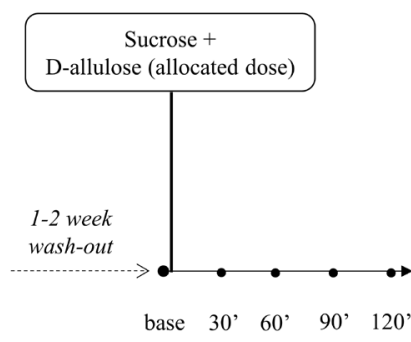

Visit 5

\section{DOSES:}

Sucrose: $50 \mathrm{~g}$

D-Allulose: either placebo or $2.5 \mathrm{~g}, 5.0 \mathrm{~g}, 7.5 \mathrm{~g}$, or $10 \mathrm{~g}$

Figure 1 Study design.

\section{Study end points, sample size and statistical analysis}

The end point of our study was the comparison between placebo and different dosing of D-allulose of (1) blood glucose and insulin concentrations at different time points, (2) blood glucose and insulin excursion and (3) area under the concentration-time curve (AUC) for blood glucose and insulin. As there were no preliminary data in Westerners, based on previous studies in Asian subjects showing that a number of 20 subjects was sufficient to detect statistically significant differences when D-allulose effect was compared with placebo, ${ }^{19}$ we planned to randomize a total of 30 subjects. This would allow for up to $25 \%$ drop out, as well as to account for higher effects of variability within our population.

For baseline characteristics, continuous variables are presented as mean $\pm \mathrm{SD}$ and categorical variables as frequency $(\%)$. Treatment effects were evaluated comparing parameters observed in the overall patient population after each dose regardless of the sequence. Comparisons of plasma glucose and insulin among doses at each time point were performed using a linear mixedeffect model with treatment group as fixed effect, and study subject as a random effect. Given the nature of the study, no adjustment for multiple comparisons was performed. Glucose and insulin excursion were calculated as the difference between the highest achieved value after sugar load and baseline. Differences in excursion among dosing was calculated with a mixedeffect model. An exploratory analysis was performed to evaluate the effects of race (white vs African-American) on D-allulose effects using a mixed model with treatment group, race and group by race interaction as fixed effects, and subject as a random effect. These results are reported as mean and $95 \% \mathrm{CI}$. AUC was measured by the trapezoidal method and compared with an analysis of variance methods. Results are reported as mean \pm SD. A p value $<0.05$ was considered to be statistically significant. Statistical analysis was performed with SPSS V.24 software (SPSS). Any adverse event reported by subjects during the study time course was recorded.

The first and last authors had full access to all data in the study and take responsibility for their integrity and the data analysis.

\section{RESULTS}

Between August 2016 and January 2017, a total of 50 subjects were identified and consented to participate in the study. Of these, 17 subjects were screen failure because of $\mathrm{HbAlc} \geq 5.8$, and 3 subjects withdrew consent before randomization. Therefore, a total of 30 subjects were randomized to study treatment. One subject withdrew consent after visit 4 , and thus data were not available for visit 5. Baseline characteristics are reported in table 1 . No serious adverse events occurred during the study time course. Two patients had minor adverse events (one patient had a vasovagal reaction after intravenous insertion and one patient reported loose stools 24 hours after sucrose plus placebo administration) that did not lead to study withdrawal.

D-allulose was associated with a dose-dependent reduction of plasma glucose at 30 min compared with placebo (across groups $\mathrm{p}=0.016$; figure 2 ). In particular, there was a trend towards reduction of plasma glucose with the 5 $\mathrm{g}$ dose $(\mathrm{p}=0.093)$, which was significantly lower with the $7.5 \mathrm{~g}$ (mean difference: 11 ; $95 \%$ CI 3 to 19 ; $\mathrm{p}=0.005$ ) 


\begin{tabular}{ll}
\hline Table 1 Baseline characteristics & \\
\hline Characteristics & $\mathbf{N}=\mathbf{3 0}$ \\
\hline Age, years & $33 \pm 8$ \\
\hline Men & $16(53 \%)$ \\
\hline Women & $14(47 \%)$ \\
\hline BMl, kg/m ${ }^{2}$ & $28 \pm 4$ \\
\hline Waist circumference (in) & $45 \pm 22$ \\
\hline Hip circumference (in) & $52 \pm 25$ \\
\hline Race & \\
\hline White & $16(53.3 \%)$ \\
\hline African-American & $5(16.7 \%)$ \\
\hline Hispanic & $4(13.3 \%)$ \\
\hline Asian & $4(13.3 \%)$ \\
\hline Other & $1(3.3 \%)$ \\
\hline Smoking & $25(83.3 \%)$ \\
\hline Never & $3(10 \%)$ \\
\hline Current & $2(6.7 \%)$ \\
\hline Former & $11(36.7 \%)$ \\
\hline Alcohol consumption & $19(63.3 \%)$ \\
\hline No &
\end{tabular}

BMI, body mass index.

and $10 \mathrm{~g}$ (mean difference: 12; 95\% CI 4 to 20; $\mathrm{p}=0.002$ ) doses compared with placebo. Although plasma glucose was not reduced at the other time points, there was a dose-dependent reduction in plasma glucose excursion compared with placebo, which was significant with the $10 \mathrm{~g}$ dose $(\mathrm{p}=0.023)$. AUC for blood glucose was similar among groups $(\mathrm{p}=0.96$; figure $3 \mathrm{~A})$.

In line with blood glucose findings, D-allulose was associated with a trend towards lower insulin levels at 30 min compared with placebo (across groups $\mathrm{p}=0.054$; figure 4), which was significant with the $10 \mathrm{~g}$ dose (mean difference: 14; $95 \%$ CI 4 to 25 ; $\mathrm{p}=0.006)$. D-alluloserelated reduction in insulin levels did not reach statistical significance at any other time point, but there was a significant dose-dependent reduction in insulin excursion compared with placebo $(\mathrm{p}=0.028)$, which was significant with the $10 \mathrm{~g}$ dose $(\mathrm{p}=0.002)$. AUC for insulin was similar among groups $(\mathrm{p}=0.40$; figure $3 \mathrm{~B})$.

The exploratory analysis comparing white $(n=16)$ and African-American $(\mathrm{n}=5)$ subjects showed no treatment by race interaction at each time point for both plasma glucose and insulin levels ( $p$ for interaction $>0.10$ for each time point) (online supplemental figures 1 and 2).

\section{DISCUSSION}

Significant reductions in the consumption of added sugars, as recommended by current guidelines, ${ }^{10-13}$ may necessitate the use of sugar substitutes to enhance food sweetness and palatability. Reduced calorie sweeteners currently approved by the FDA include sugar alcohols (polyols) such as erythritol, isomalt, lactitol, maltitol, mannitol, sorbitol, xylitol and hydrogenated starch hydrolysates. Studies of subjects with and without DM have shown that sugar alcohols produce a lower postprandial glucose response than sucrose or glucose and

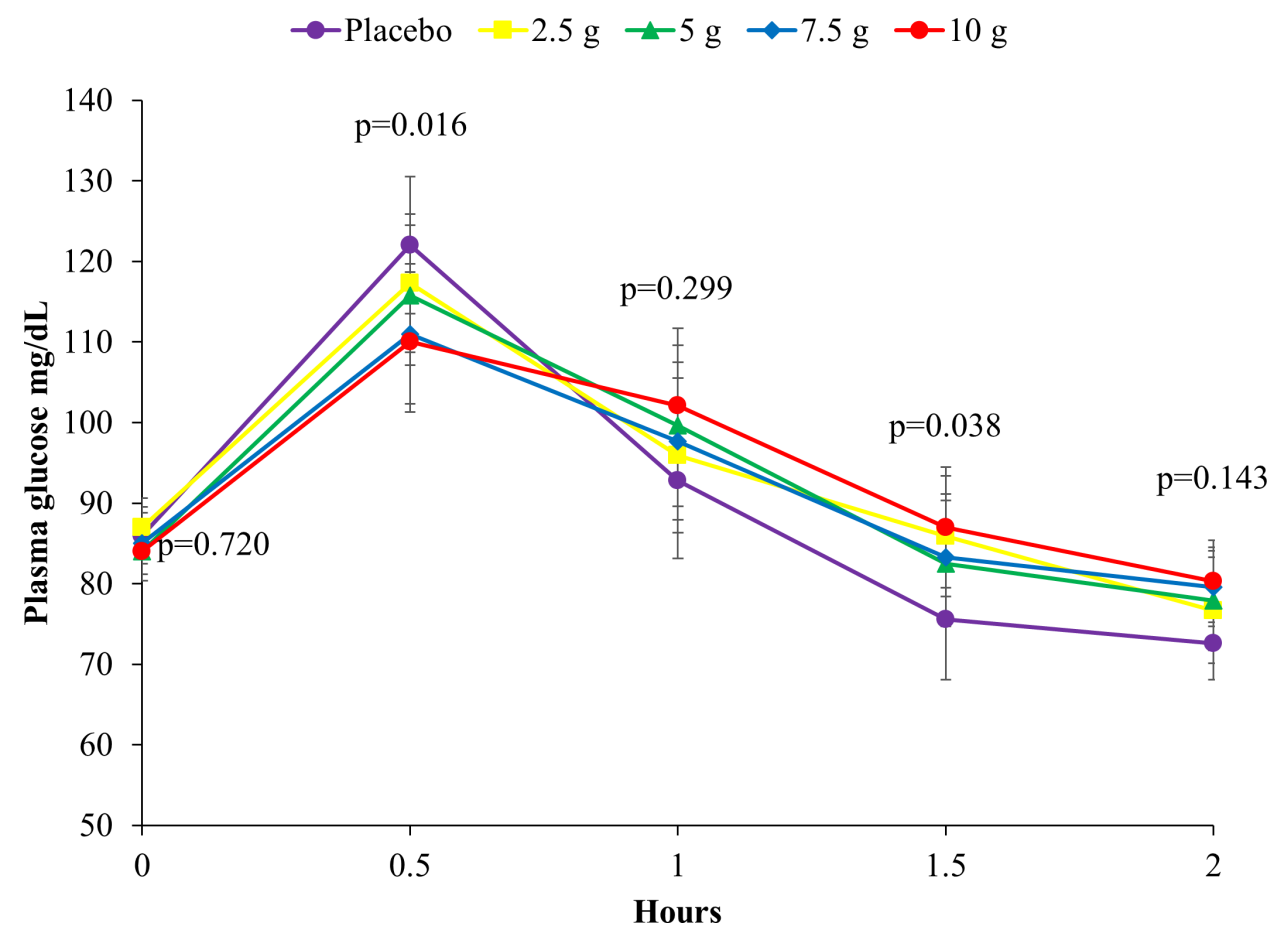

Figure 2 Comparison of plasma glucose levels among placebo and different D-allulose doses. Results are reported as mean. Error bars represent $95 \% \mathrm{Cl}$. P values represent the overall comparisons among doses at each time point. 


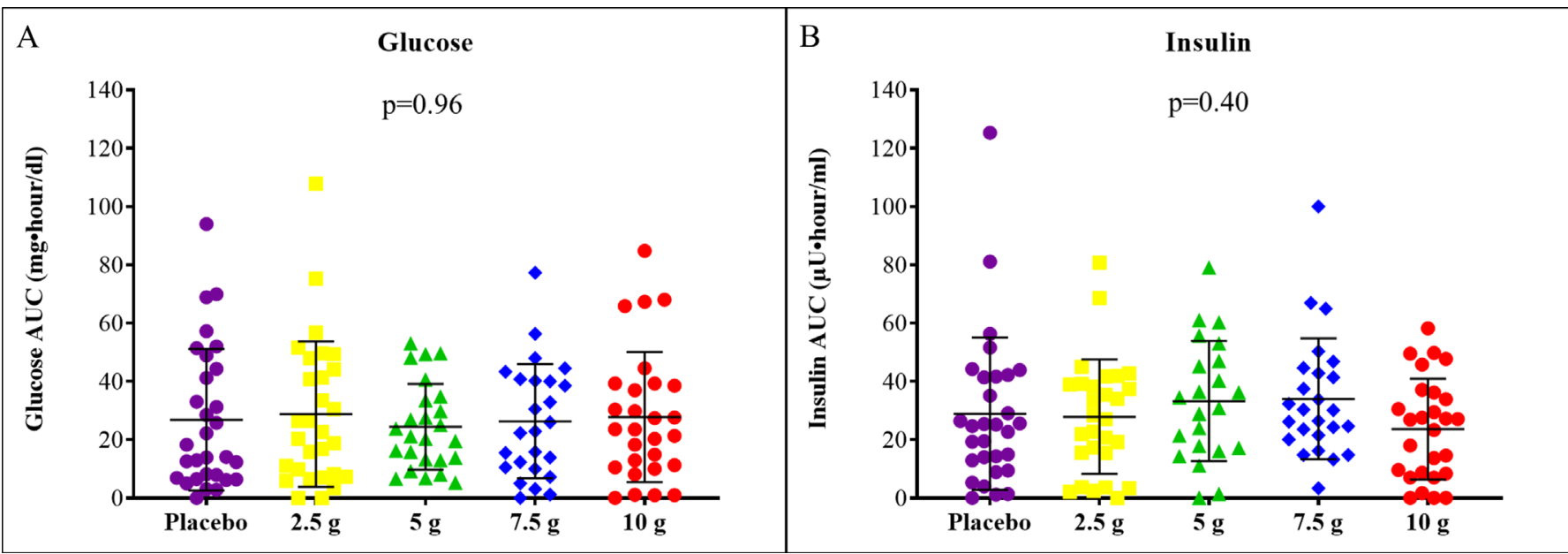

Figure 3 Comparison of plasma glucose and insulin area under the concentration-time curve (AUC) among placebo and different $D$-allulose doses. AUC for blood glucose $(A)$ and insulin $(B)$ according to different $D$-allulose doses. $P$ values represent the overall comparisons among doses. Results are reported as individual values; bars indicate mean \pm SD.

have lower available energy. ${ }^{14}{ }^{15}$ Sugar alcohols contain, on average, about 2 calories/g (one-half the calories of other sweeteners such as sucrose). Use of sugar alcohols as sweeteners reduces the risk of dental caries. However, there is no evidence that the amounts of sugar alcohols likely to be consumed will reduce glycemia, energy intake or body weight. The use of sugar alcohols appears to be safe; however, they may cause diarrhea. The FDA has approved six non-nutritive sweeteners for use in the USA. These are acesulfame potassium, aspartame, neotame, saccharin, stevia and sucralose. Clinical studies involving subjects without diabetes provide no indication that non-nutritive sweeteners in foods will cause weight loss or weight gain..$^{14}$

Rare sugar D-allulose as a substitute sweetener is produced through the isomerization of $\mathrm{D}$-fructose by D-tagatose 3-epimerases or D-allulose 3-epimerases. It is a low energy monosaccharide sugar naturally existing in very small quantities in some fruits, including kiwis (9.4 $\mathrm{mg} / 100 \mathrm{~g}$ food), figs (29.6 mg/100 $\mathrm{g}$ food) and raisins $(38.7 \mathrm{mg} / 100 \mathrm{~g}$ food $){ }^{15}$ D-allulose has approximately $70 \%$ relative sweetness to sucrose with $0.2 \mathrm{kcal} / \mathrm{g}$ that is a $95 \%$ calorie reduction compared with sucrose. ${ }^{16}$ With the advent of technology that allows the production of

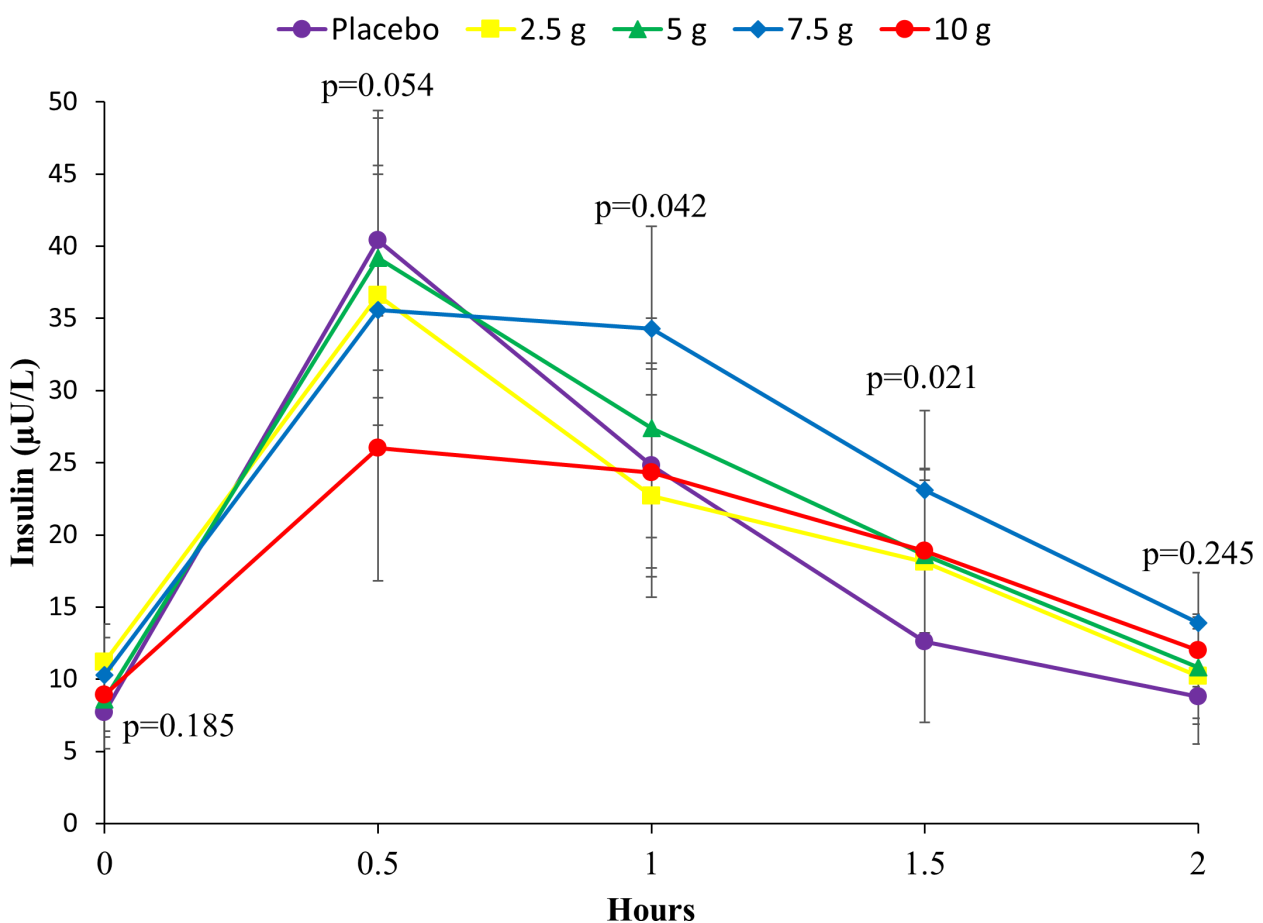

Figure 4 Comparison of insulin levels among placebo and different D-allulose doses. Results are reported as mean. Error bars represent $95 \% \mathrm{Cl}$. P values represent the overall comparisons among doses at each time point. 
D-allulose enzymatically on a large scale, ${ }^{28}{ }^{29}$ it is now possible to conduct studies of utility of D-allulose in food and beverages. D-Allulose possesses high value as a food ingredient and dietary supplement, and may carry a variety of physiological functions, such as improving insulin resistance, antioxidant enhancement, reducing endoplasmic reticulum stress and hyperglycemic control, which makes it a very attractive option. However, so far, glucose suppressive effects of D-allulose have been mostly shown in animal studies and small clinical studies performed in Asian populations, ${ }^{17-20}$ and only limited data exist in Westerners. ${ }^{21} 22$

Our study was the largest to test the effects of D-allulose in Westerners, including white and African-American, and results can be summarized as follows: (1) D-allulose administered in addition to a standard sucrose load led to a dose-dependent reduction of plasma glucose at $30 \mathrm{~min}$ compared with placebo; (2) although plasma glucose was not reduced at the other time points and AUC was similar between D-allulose and placebo, there was a dose-dependent reduction in plasma glucose excursion compared with placebo; (3) the effects of D-allulose on insulin levels and insulin excursion were similar to those on plasma glucose; (4) D-allulose effects appeared to be consistent in both white and African-American subjects; (5) the administration of escalating doses of D-allulose appeared to be safe.

Various physiological activities of D-allulose have been reported so far. Of those, its postprandial glucose suppressive effect has been proven by both animal and clinical studies. ${ }^{17-20}$ Several animal experiments have reported antiobesity and antidiabetic effects of D-allulose. ${ }^{23-27}$ There are multiple potential mechanisms that contribute to the antiglycemic effect of D-allulose. In one study, the antiglycemic effect of D-allulose was attributed to its ability to increase glucagon-like peptide 1 levels. ${ }^{30}$ Shintani et al attributed the improved glucose tolerance and insulin sensitivity in Wistar rats to changes in hepatic glucokinase. ${ }^{31}$ Another study in animals suggested that the postprandial glucose suppression could be at least partly related to the inhibition of intestinal $\alpha$-glycosidase. ${ }^{17}$ In this latter study, D-allulose was also shown to suppress hepatic lipogenic enzyme activity and reduce intra-abdominal fat accumulation. Overall, it appears that the effect of D-allulose is independent of insulin and the changes in insulin concentrations during these experiments are secondary to changes in plasma glucose.

In small clinical trials conducted in Japan, D-allulose significantly suppressed blood glucose elevation after glucose loading study on healthy adults. ${ }^{19} 20$ These studies also suggested that supplemental D-allulose in the diet could reduce postprandial glycemic response and might have antidiabetic effects. In addition, studies on rats and humans did not detect any increase in carbohydrate energy expenditure by D-allulose absorbed through the small intestine, even at the upper limit of concentration. ${ }^{17-20}$ Finally, clinical studies have also shown that D-allulose does not affect blood glucose or insulin levels after ingestion, ${ }^{19}$ and in a randomized, double-blind, controlled trial of long-term ingestion of D-allulose 3 times a day with meal for 12 weeks in healthy human subjects did not show any abnormal effects or clinical problems. ${ }^{20}$

Early clinical trials of D-allulose demonstrating its antidiabetic and antiobesity effects have been carried out in Kagawa (Japan). In 2011, a rare sugar syrup (RSS), which contains $76 \%$ of regular syrup (D-glucose and D-fructose), $6 \%$ of D-allulose and $18 \%$ of other saccharides including D-allose and D-sorbose, has been developed and commercialized in Japan and D-allulose and D-allulose-containing RSS have already been used for various foods. ${ }^{32}$ Two small studies conducted in subjects with or without DM in Canada showed none or modest reductions in the postprandial blood glucose response to oral glucose with administration of D-allulose. ${ }^{21} 22$ Our investigation is the largest conducted so far in Westerners, including both white and African-American, and using multiple D-allulose dosing regimens. The results of our investigation, which confirms D-allulose properties in reducing postprandial glycemic response, as well as safety, among Westerners, can set the basis for the potential use of $\mathrm{D}$-allulose in a population where reduction of added sugar is of paramount importance. The results of the present study, along with the results in previously published studies, suggest that adding 5-10 g of D-allulose to $50 \mathrm{~g}$ of sugar is sufficient to reduce the postprandial glycemia attributed to sugar. This ratio of D-allulose-to-sugar content is consistent with the composition of the currently marketed RSS, which contains only $6 \%$ of D-allulose and is well tolerated. Thus, the amount of D-allulose that would be necessary for an effect and that is reasonable in food products is $5 \%-10 \%$ of added table sugar.

It is noteworthy that targeting postprandial blood glucose spike may have beneficial clinical effects independent of the average blood glucose levels as estimated by $\mathrm{HbA1c}$ measurements. ${ }^{33}{ }^{34}$ In endothelial cell cultures there is rapid adaptive downregulation of oxidative burst induced by high concentrations of dextrose suggesting that repeated spikes of blood glucose levels are more likely to cause oxidative damage than sustained levels of blood glucose. ${ }^{35}$ Future studies should explore more in depth the underlying mechanisms by which $\mathrm{D}$-allulose reduces glucose and insulin levels as well as its long-term effects, including its potential to impact clinically meaningful outcomes.

\section{Study limitations}

Our study was of short duration and used a single administration of D-allulose in any given day of testing, therefore the effects and safety of long-term administration were not assessed and would require dedicated studies. It is in fact pivotal to identify the potential negative effects of administered rare sugars. For example, trehalose, a rare disaccharide, was found to enhance Clostridium difficile disease. ${ }^{36}$ We did not study the effects of doses 
of D-allulose higher than $10 \mathrm{~g}$. Although our dosing is in line with previously reported studies in Asians, we cannot rule out the possibility that higher doses would be more effective and well tolerated in Western populations. Our study was not powered to detect differences in treatment according to race, and included a limited number of African-American subjects. Therefore, our results showing consistent treatment effects in white and African-American subjects should be interpreted with caution and considered as hypothesis generating.

\section{CONCLUSIONS}

This is the first study assessing the effects of D-allulose in a Western population demonstrating a dose-dependent reduction in postprandial plasma glucose and insulin levels as well as decreased postprandial glucose and insulin excursion in subjects without DM. These pilot observations set the basis for large-scale investigations to help support the anti-DM effects of D-allulose.

Contributors FF, DMY, ADM and DJA designed the study protocol and drafted the manuscript. FF designed the statistical analyses and analyzed the data. LB, DMY, $F R, A R$ and JRR were involved in patient recruitment. LB, DMY, FR, AR, JRR YT, MT, $\mathrm{TI}$ and $\mathrm{NH}$ critically revised the manuscript for important intellectual content. All authors approved the final manuscript.

Funding The study was funded by an investigator-initiated grant from Matsutani Chemical Industry Co. Ltd. Matsutani Chemical Industry Co. Ltd. had no role in study design conception, conduct of the study or decision to publish these results.

Competing interests FF has received payment as an individual for consulting fee or honorarium from AstraZeneca, Bayer and Sanofi. DJA has received payment as an individual for: (a) consulting fee or honorarium from Abbott, Amgen, Aralez, AstraZeneca, Bayer, Biosensors, Boehringer Ingelheim, Bristol-Myers Squibb, Chiesi, Daiichi-Sankyo, Eli Lilly, Haemonetics, Janssen, Merck, PhaseBio, PLx Pharma, Pfizer, Sanofi and The Medicines Company; (b) participation in review activities from CeloNova and St. Jude Medical. Institutional payments for grants from Amgen, AstraZeneca, Bayer, Biosensors, CeloNova, CSL Behring, DaiichiSankyo, Eisai, Eli-Lilly, Gilead, Idorsia, Janssen, Matsutani Chemical Industry Co. Ltd., Merck, Novartis, Osprey Medical, Renal Guard Solutions and the Scott R. MacKenzie Foundation. YT, $\mathrm{Tl}$ and $\mathrm{NH}$ are employees of Matsutani Chemical Industry Co. Ltd., which is a manufacturer of D-allulose. All other authors have no conflicts of interest to disclose.

Patient consent for publication Not required.

Ethics approval This study was approved by the Western Institutional Review Board (protocol number 20160589) and complied with the Declaration of Helsinki. All subjects gave their written informed consent.

Provenance and peer review Not commissioned; externally peer reviewed.

Data availability statement All data relevant to the study are included in the article or uploaded as supplemental information.

Supplemental material This content has been supplied by the author(s). It has not been vetted by BMJ Publishing Group Limited (BMJ) and may not have been peer-reviewed. Any opinions or recommendations discussed are solely those of the author(s) and are not endorsed by BMJ. BMJ disclaims all liability and responsibility arising from any reliance placed on the content. Where the content includes any translated material, BMJ does not warrant the accuracy and reliability of the translations (including but not limited to local regulations, clinical guidelines, terminology, drug names and drug dosages), and is not responsible for any error and/or omissions arising from translation and adaptation or otherwise.

Open access This is an open access article distributed in accordance with the Creative Commons Attribution Non Commercial (CC BY-NC 4.0) license, which permits others to distribute, remix, adapt, build upon this work non-commercially, and license their derivative works on different terms, provided the original work is properly cited, appropriate credit is given, any changes made indicated, and the use is non-commercial. See: http://creativecommons.org/licenses/by-nc/4.0/.
ORCID iD

Francesco Franchi http://orcid.org/0000-0001-8503-5736

\section{REFERENCES}

1 Bessesen DH. Update on obesity. J Clin Endocrinol Metab 2008;93:2027-34.

2 American Diabetes Association, Bantle JP, Wylie-Rosett J, et al. Nutrition recommendations and interventions for diabetes: a position statement of the American diabetes association. Diabetes Care 2008;31 Suppl 1:S61-78.

3 Wylie-Rosett J, Albright AA, Apovian C, et al. 2006-2007 American diabetes association nutrition recommendations: issues for practice translation. J Am Diet Assoc 2007;107:1296-304.

4 Mead A, Atkinson G, Albin D, et al. Dietetic guidelines on food and nutrition in the secondary prevention of cardiovascular disease? evidence from systematic reviews of randomized controlled trials (second update, January 2006). J Hum Nutr Diet 2006;19:401-19.

5 Hession M, Rolland C, Kulkarni U, et al. Systematic review of randomized controlled trials of low-carbohydrate vs. low-fat/lowcalorie diets in the management of obesity and its comorbidities. Obes Rev 2009;10:36-50.

6 Mooradian AD. The merits and the pitfalls of low carbohydrate diet: a Concise review. J Nutr Health Aging 2020;24:805-8.

7 Barclay AW, Petocz P, McMillan-Price J, et al. Glycemic index, glycemic load, and chronic disease risk--a meta-analysis of observational studies. Am J Clin Nutr 2008;87:627-37.

8 Palmer JR, Boggs DA, Krishnan S, et al. Sugar-Sweetened beverages and incidence of type 2 diabetes mellitus in African American women. Arch Intern Med 2008;168:1487-92.

9 Malik VS, Popkin BM, Bray GA, et al. Sugar-Sweetened beverages and risk of metabolic syndrome and type 2 diabetes: a metaanalysis. Diabetes Care 2010;33:2477-83.

10 Johnson RK, Appel LJ, Brands M. American heart association nutrition Committee of the Council on nutrition, physical activity, and metabolism and the Council on epidemiology and prevention. dietary sugars intake and cardiovascular health: a scientific statement from the American heart association. Circulation 2009;120:1011-20.

11 The National Acadamies Sciences Engineering Medicine. Dietary reference intakes for energy, carbohydrate, fiber, fat, fatty acids, cholesterol, protein, and amino acids. National academies press web site, 2020. Available: http://www.nap.edu/openbook.php?record id= 10490

12 CDC. CDC guidelines on added sugars consumption, 2020 Available: https://www.cdc.gov/nutrition/data-statistics/know-yourlimit-for-added-sugars.html

13 Dietary Guide Lines. The 2015-2020 dietary guidelines for Americans, 2020. Available: https://health.gov/sites/default/files/ 2019-10/DGA_Cut-Down-On-Added-Sugars.pdf

14 Mooradian AD, Smith M, Tokuda M. The role of artificial and natura sweeteners in reducing the consumption of table sugar: a narrative review. Clin Nutr ESPEN 2017:18:1-8.

15 Mooradian AD. In search for an alternative to sugar to reduce obesity. Int J Vitam Nutr Res 2019;89:113-7.

16 Cree GM, Perlin AS. O-isopropylidene derivatives of d-allulose (dPsicose) and D-erythro-hexopyranos-2,3-diulose. Can J Biochem 1968;46:765-70.

17 Matsuo T, Izumori K. D-Psicose inhibits intestinal alpha-glucosidase and suppresses the glycemic response after ingestion of carbohydrates in rats. J Clin Biochem Nutr 2009;45:202-6.

18 Matsuo T, Suzuki H, Hashiguchi M, et al. D-Psicose is a rare sugar that provides no energy to growing rats. J Nutr Sci Vitaminol 2002;48:77-80.

19 lida T, Kishimoto Y, Yoshikawa Y, et al. Acute D-psicose administration decreases the glycemic responses to an oral maltodextrin tolerance test in normal adults. J Nutr Sci Vitaminol 2008;54:511-4

20 Hayashi N, lida T, Yamada T, et al. Study on the postprandial blood glucose suppression effect of D-psicose in borderline diabetes and the safety of long-term ingestion by normal human subjects. Biosci Biotechnol Biochem 2010;74:510-9.

21 Braunstein CR, Noronha JC, Glenn AJ, et al. A double-blind, randomized controlled, acute feeding equivalence trial of small, catalytic doses of fructose and allulose on postprandial blood glucose metabolism in healthy participants: the fructose and allulose catalytic effects (face) trial. Nutrients 2018;10:750.

22 Noronha JC, Braunstein CR, Glenn AJ, et al. The effect of smal doses of fructose and allulose on postprandial glucose metabolism in type 2 diabetes: a double-blind, randomized, controlled, acute feeding, equivalence trial. Diabetes Obes Metab 2018;20:2361-70. 
23 Hossain A, Yamaguchi F, Matsunaga T, et al. Rare sugar D-psicose protects pancreas $\beta$-islets and thus improves insulin resistance in OLETF rats. Biochem Biophys Res Commun 2012;425:717-23.

24 Ochiai M, Onishi K, Yamada T, et al. D-Psicose increases energy expenditure and decreases body fat accumulation in rats fed a highsucrose diet. Int J Food Sci Nutr 2014;65:245-50.

25 Matsuo T, Izumori K. Effects of dietary D-psicose on diurnal variation in plasma glucose and insulin concentrations of rats. Biosci Biotechnol Biochem 2006;70:2081-5.

26 Ochiai M, Nakanishi Y, Yamada T, et al. Inhibition by dietary Dpsicose of body fat accumulation in adult rats fed a high-sucrose diet. Biosci Biotechnol Biochem 2013;77:1123-6.

27 Chung Y-M, Hyun Lee J, Youl Kim D, et al. Dietary D-psicose reduced visceral fat mass in high-fat diet-induced obese rats. $J$ Food Sci 2012;77:H53-8.

28 Takeshita K, Suga A, Takada G, et al. Mass production of Dpsicose from D-fructose by a continuous bioreactor system using immobilized D-tagatose 3-epimerase. J Biosci Bioeng 2000;90:453-5.

29 Granström TB, Takata G, Tokuda M, et al. Izumoring: a novel and complete strategy for bioproduction of rare sugars. J Biosci Bioeng 2004;97:89-94.
30 Iwasaki Y, Sendo M, Dezaki K, et al. Glp-1 release and vagal afferent activation mediate the beneficial metabolic and chronotherapeutic effects of d-allulose. Nat Commun 2018;9:113.

31 Shintani T, Yamada T, Hayashi N, et al. Rare sugar syrup containing d-allulose but not high-fructose corn syrup maintains glucose tolerance and insulin sensitivity partly via hepatic glucokinase translocation in Wistar rats. J Agric Food Chem 2017;65:2888-94.

32 Hayashi N, Yamada T, Takamine S, et al. Weight reducing effect and safety evaluation of rare sugar syrup by a randomized double-blind, parallel-group study in human. J Funct Foods 2014;11:152-9.

33 Mooradian AD, Thurman JE. Drug therapy of postprandial hyperglycaemia. Drugs 1999;57:19-29.

34 Aryangat AV, Gerich JE. Type 2 diabetes: postprandial hyperglycemia and increased cardiovascular risk. Vasc Health Risk Manag 2010;6:145-55.

35 Horani MH, Haas MJ, Mooradian AD. Rapid adaptive down regulation of oxidative burst induced by high dextrose in human umbilical vein endothelial cells. Diabetes Res Clin Pract 2004;66:7-12.

36 Collins J, Robinson C, Danhof H, et al. Dietary trehalose enhances virulence of epidemic Clostridium difficile. Nature 2018;553:291-4. 